\title{
Social Information Retrieval and Recommendation: state- of-the-art and future research
}

\author{
Abir Gorrab, Ferihane Kboubi and Henda Ben Ghezala \\ RIADI Laboratory-ENSI \\ University of Manouba \\ Manouba 2010, Tunisia \\ Abir.Gorrab@riadi.rnu.tn

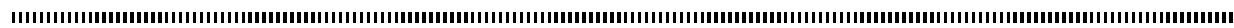

ABSTRACT. The explosion of web 2.0 and social networks has created an enormous and rewarding source of information that has motivated researchers in different fields to exploit it. Our work revolves around the issue of access and identification of social information and their use in building a user profile enriched with a social dimension, and operating in a process of personalization and recommendation. We study several approaches of Social IR (Information Retrieval), distinguished by the type of incorporated social information. We also study various social recommendation approaches classified by the type of recommendation. We then present a study of techniques for modeling the social user profile dimension, followed by a critical discussion. Thus, we propose our social recommendation approach integrating an advanced social user profile model.

RÉSUMÉ. L'explosion du web 2.0 et des réseaux sociaux a crée une source d'information énorme et enrichissante qui a motivé les chercheurs dans différents domaines à l'exploiter. Notre travail s'articule autour de la problématique d'accès et d'identification des informations sociales et leur exploitation dans la construction d'un profil utilisateur enrichi d'une dimension sociale, et son exploitation dans un processus de personnalisation et de recommandation. Nous étudions différentes approches sociales de RI (Recherche d'Information), distinguées par le type d'informations sociales incorporées. Nous étudions également diverses approches de recommandation sociale classées par le type de recommandation. Nous exposons ensuite une étude des techniques de modélisation de la dimension sociale du profil utilisateur, suivie par une discussion critique. Ainsi, nous présentons notre approche de recommandation sociale proposée intégrant un modèle avancé de profil utilisateur social.

KEYWORDS: social information retrieval, social recommendation, social networks, user profile

MOTS-CLÉS : recherche d'information sociale, recommandation sociale, réseaux sociaux, profil utilisateur. 


\section{Introduction}

The apparition of the social web and the explosion of social networks have revolved the web in a measure that users become able not only to consume, but also to product informational content. As a matter of fact, the huge number of web users and time spent daily on internet motivated researchers in IR and encouraged them to benefit from this content as an enlightening source of information. Besides, social networks and collaborative sites (such as Facebook, LinkedIn, Google+, Twitter, YouTube, Delicious, CiteUlike, etc) are the most common and popular source of interactive content. The number of users of these networks is growing unconditionally and the number of their active users is very high.

In this paper, we focus on the impact of social information integration in an IR process and a recommendation system by presenting an overview of social IR and recommendation works. We present also our social recommender system including an enhanced social user profile model.

The remainder of this paper is organized as follows. Section 2 presents the main approaches used in Social IR. Section 3 then describes social recommendation works, and section 4 is reserved to social dimension in user profile modeling. In section 5, we discuss those works and identify future research challenges. Section 6 is dedicated to the presentation of our proposed recommender system incorporating an enhanced social user profile model. Finally, section 7 draws conclusions and future directions.

\section{Social IR approaches}

Social Information Retrieval is a topical field that aims at integrating social informational resources in the research process. Social IR approaches are various. They are mostly based on social information identification and integration in a search process. In fact, several types of social information are used in social IR works. We can cite tags, social relations, comments, tweets, like and dislike mentions, folksonomies, conversations, hashtags, shares, etc. 
Proposed approaches widely use many social information, which can be integrated in different levels in IR process: user profile construction, query expansion and result ponderation. Below we distinguish social IR approaches, based on the specific social information taken into account.

\subsection{Approach based on social annotations and relations}

Social annotations are a valuable informational source that enhances social IR by including user's area of interest. Bouhini et al. [2] propose a user profile generation approach from folksonomies. This work combines queries with user profile based on terms frequency. It presents two Social IR models inspired from BM25 model which are BM25S Score Comb and BM25S Freq Comb. These models combine query and user profile using respectively scores and terms frequency. PengLi et al [30] propose a TRLDA model of annotations categorization. This work introduces representation and ponderation methods of annotation categories. In fact, authors study the effect of annotations' incorporation in IR process. Moreover, Bao et al. [34] calculate similarity between web query and social annotations. They propose two algorithms that enhance web IR: SPR (Social Page Rank) that estimates web pages popularity and SSR (Social Sim Rank) that computes similarity degree.

Furthermore, users may be linked by different relationships that are specific to each social network. They can be followers, friends, co-authors or even belonging to the same group on a social network. Works based on this approach usually use this informational content generated by relations, by combining a social and a thematic score. Moreover, Amer et al. [28] propose a probabilistic model that indexes conversation indexation in twitter. This model exploits social relations to measure users' activity, influence and expertise. In this context, Ben Jabeur et al. [4] propose a social model based on Bayesian network that measures two social relevance factors which are User social importance, evaluated by a PageRank score; and the number of temporal neighbors.

\subsection{Approach based on social signals}

Shared statuses, comments, like and dislike mentions are considered as social signals and are being more explored in social IR researches, seeing the relevant information they bring. In this issue, Chelaru et al [9] study the impact of these social signals in video search on YouTube, by combining social information such as comments, like and dislike mentions, with basic search criteria (similarity between the query and video title), which enhances the performance of videos' extraction process. Furthermore, Badache et al. [3] investigate on a language model incorporating temporal characteristics of social signals (number of like mentions, shares and comments) to estimate resources' relevance and sort search results. Moreover, Ramesh et al. [33] examine the personalized social IR 
process and propose a user profile construction algorithm exploiting pages liked on Facebook, through different user's accounts. This social content personalizes search results.

\subsection{Comparative study}

To discuss and compare Social IR approaches, we led a comparative study of different works based on several categories. For each work we considered the following six points as a comparative criteria: (1) the social network used for the experimentation, (2) the techniques used in the presented models, (3) the metrics used for the evaluation, (4) if there is a CI (Combination of Information), (5) if there is a CSN (Combination of Social Networks) and (6) if the work considered the TA (Temporal Aspect). Table 1 summarizes the results of our study. 


\begin{tabular}{|c|c|c|c|c|c|c|c|}
\hline \multicolumn{2}{|c|}{$\begin{array}{l}\text { Social } \\
\text { information }\end{array}$} & \multirow[t]{2}{*}{$\begin{array}{l}\text { Social } \\
\text { network }\end{array}$} & \multirow{2}{*}{$\begin{array}{l}\text { Techniques } \\
\begin{array}{l}\text { BM25and } \\
\text { derivatives }\end{array}\end{array}$} & \multirow{2}{*}{$\begin{array}{l}\text { Evaluation } \\
\text { MAP, P[0,1] }\end{array}$} & \multirow{2}{*}{$\begin{array}{r}\mathbf{C I} \\
-\end{array}$} & \multirow{2}{*}{$\begin{array}{r}\mathbf{C S N} \\
-\end{array}$} & \multirow{2}{*}{$\begin{array}{r}\text { TA } \\
-\end{array}$} \\
\hline $\begin{array}{l}\text { Annota } \\
\text { ti-ons }\end{array}$ & {$[2]$} & & & & & & \\
\hline & [34] & Del.ic.ious & SSR, SPR & $\begin{array}{l}\text { MAP, } \\
\text { nDCG }\end{array}$ & - & - & - \\
\hline & {$[30]$} & Del.ic.ious & $\begin{array}{l}\text { TF_IDF, } \\
\text { Inference } \\
\text { algorithm }\end{array}$ & - & - & - & - \\
\hline \multirow[t]{2}{*}{$\begin{array}{l}\text { Social } \\
\text { relatio } \\
\text { ns }\end{array}$} & {$[4]$} & $\begin{array}{l}\text { Twitter, } \\
\text { Citulike }\end{array}$ & $\begin{array}{l}\text { PageRank, } \\
\text { language model, } \\
\text { ImpG:social } \\
\text { score, } \\
\text { TF-IDF }\end{array}$ & MAP, recall & $\sqrt{ }$ & $\sqrt{ }$ & $\sqrt{ }$ \\
\hline & [28] & Twitter & $\begin{array}{l}\text { BM25, language } \\
\text { model, } \\
\text { PageRank }\end{array}$ & $\begin{array}{l}\text { Leave One } \\
\text { Out } \\
\text { approach, } \\
\text { MAP }\end{array}$ & $\sqrt{ }$ & - & - \\
\hline \multirow[t]{3}{*}{$\begin{array}{l}\text { Social } \\
\text { signals }\end{array}$} & [9] & Youtube & $\begin{array}{l}\text { TF_IDF, } \\
\text { Lucene, } \\
\text { SentiWord-Net }\end{array}$ & Ndcg & $\sqrt{ }$ & - & - \\
\hline & {$[3]$} & $\begin{array}{l}\text { Facebook, } \\
\text { Twitter, } \\
\text { LinkedIn, } \\
\text { Del.ic.ious, } \\
\text { Google+ }\end{array}$ & Language model & $\begin{array}{l}\text { MAP, } \\
\text { nDCG, } \\
\text { Recall, } \\
\text { Precision }\end{array}$ & $\sqrt{ }$ & $\sqrt{ }$ & $\sqrt{ }$ \\
\hline & [19] & Facebook & $\begin{array}{l}\text { Clustering data } \\
\text { TF-IDF }\end{array}$ & $\begin{array}{l}\text { Performance } \\
\text { measure }\end{array}$ & $\sqrt{ }$ & - & - \\
\hline
\end{tabular}

Table1. Comparative table of Social IR approaches categorized by social information types

In table 1, we present a comparative study of some works related to the several approaches described in this section, based on annotations, social relations and social signals. We studied the aspects of the combination of many social information or social networks and the consideration of temporal aspect. These features enhance IR processes 
and improve their performances. In fact, many networks are used and many techniques are conducted, but temporal aspect and the combination of different networks represent the greatest motivation for researchers.

\section{Social recommendation}

Social recommendation is a set of methods that try to suggest items or entities that seem to be interesting to the user, using his social information [12]. In fact, there are three main recommendation techniques [5] [25] [13]: content based approach, collaborative filtering and hybrid technique. In content based approach, the user is provided with entities that are similar to those he has chosen in the past. On the other hand, recommender systems based on collaborative filtering suggest entities that are similar to other people's choice, having similar preferences. Meanwhile, hybrid recommender systems combine both content-based and collaborative filtering techniques, so as to enhance recommendations' quality.

Several methods have been proposed to recommend relations and entities, like Trust Walker [27], Mole Trust [31], Social MF [26] and SoRec [37] for items recommendation, which are collaborative filtering techniques. There are also multiple scores of similarity calculation, like SimRank, Jaccard coefficient and Katz.

Additionally, many researchers have explored social information to improve recommender systems. Notably, Hafsi et al. [18] exploit user-generated content (rating and review) in books recommendation system. Their work measures books reputation and popularity concepts and tests three approaches: book tags and reviews indexation, themes interrogation and users similarity calculation. Unlike in [7], authors have proposed a content-based approach that compares user profiles' information in order to determine similarities between them and recommend friendship relations. On the other hand, Wang et al. [39] investigate on tag based social recommendation by calculating tags' similarities and connecting users that are likely to have similar tastes and preferences. In the same context, Hannon et al. [19] propose an hybrid recommendation system using content and collaborative-based approaches that recommends users to follow in the social network Twitter, by analyzing their profiles.

Friendship relations are also suggested in [7] in a content based approach, by comparing Facebook and MySpace user profiles and calculating profiles' similarities, using Content Matching and Friend of Friend algorithms. Furthermore, a social recommendation system based on friend circles is proposed in [40]. This approach estimates trust between users and constructs presumed circles to use in recommendation process. 
Temporal aspect of social information features is a rewarding and relevant social resource that enhances social recommendation process. In this context and for location recommendation purposes, a recommendation framework is proposed in [20]. This work follows users' movement temporal properties and introduces temporal aggregation strategies, in order to take into account users' preferences in different temporal states. In [24], a session-based temporal graph modeling two user preferences types is presented: long term and short term preferences are merged, and the paper models their interaction, so as to have more information about the user.

In the following, we conduct a comparative table of several social recommendation works evolving the techniques used in each work and also the social information adopted.

\begin{tabular}{|c|c|c|c|c|c|}
\hline Works & Techniques & $\begin{array}{l}\text { Social } \\
\text { signals }\end{array}$ & $\begin{array}{l}\text { Temporal } \\
\text { features }\end{array}$ & $\begin{array}{l}\text { Positive } \\
\text { relations }\end{array}$ & $\begin{array}{l}\text { Negative } \\
\text { features }\end{array}$ \\
\hline$[18]$ & BM25 & + & - & - & - \\
\hline [7] & $\begin{array}{l}\text { CONTENT MATCHING/ } \\
\text { FRIEND OF FRIEND } \\
\text { ALGORITHMS }\end{array}$ & + & - & - & - \\
\hline [39] & DIFFUSION KERNELS & + & - & - & - \\
\hline [19] & LUCENE/ TF-IDF & + & - & + & - \\
\hline$[40]$ & $\begin{array}{l}\text { PRESUMED TRUST } \\
\text { CIRCLES }\end{array}$ & + & - & - & - \\
\hline$[20]$ & $\begin{array}{l}\text { AGGREGATION } \\
\text { STRATEGIES }\end{array}$ & + & + & - & - \\
\hline [24] & GRAPH MODELING & + & + & - & - \\
\hline
\end{tabular}

Table2. Comparative table of Social Recommendation approaches 


\section{Social dimension in user profile modeling}

User profile modeling is an essential task in Personalized IR. This entity brings and organizes the information necessary to define the user and describe his interests.

Following the emergence of social networks, Social IR has widely evolved. Thus, the social dimension of the user profile has become an essential component in social personalization systems. A lot of works were directed towards the construction of a social profile based on annotations [17] [38], given the importance of the data they generate. Others have focused on the analysis of egocentric social network, they are interested in friendship relationships in social networks [11] [8]. This information produces relevant content for collaboration within social IR systems. It solves the cold start problem, or lack of user's activity on social networks. The temporal aspect is also reflected in some works [8], which differentiates between recent and old social activities, to estimate their importance. Other social signals have also been integrated into the social dimension of user profile such as comments and shares. Once the profiles are built, some authors have thought of building virtual communities of users, based on similarity degree between the profiles. These communities are considered as a dimension in the profile. They are very rewarding and provide additional relevant information. In [10], Dridi et al model a user profile based on annotations and exploit it to detect communities based on annotations' similarities. For community detection, Katz index is used. It calculates the similarity taking into account the direct and indirect links in a graph. Moreover, [36] presents item recommendation approach that represents users and items' profiles using social signals, then calculates profiles' similarities using BM25 and TF-IDF [14] techniques. Addressing the same issue, Leili et al [23] proposed an hybrid news recommendation system that models the temporal evolution of user's interest. A long term and short term user profile is constructed. Long term user profile models general interests, while short term profile includes recent and current preferences.

\section{Discussion}

In this section, we discuss different aspects related to research in Social IR and recommendation topics. In fact, classical approaches in these fields do not take into account user's social content provided by his interactions and social relations. Moreover, most of the existing social approaches use social signals, tags or relational information. Some works started leveraging different types of information. Also, combining social content from many social networks and matching different user's social profiles improve the collection of relevant information that better describe the user and enhance his affluence. 
The construction of a data collection relative to Social IR or recommender systems is basically a major challenge. For this issue, we led a technical study of a set of social networks API that are likely to be the most known. Some social networks don't present yet API for developers, like ResearchGate. In the extraction process, the majority of social networks use the OAuth 2.0 for connection and authentication authority, like Twitter, Youtube, Google+, LinkedIn, and Foursquare. Delicious and CiteUlike require basic http authentication, while some other networks need API keys for authentication (Last.fm, Flickr). REST is the common API used to have access to resources, and the result is always a JSON or XML file. Actually, this study is our way to construct a data collection suitable for Social IR.

Temporality is a fundamental issue and the most central aspect in social content. This factor is being investigated in several works [8][7][18] but still presents new contribution areas. Temporal aspect supports the eventual and permanent evolution of users' tastes, preferences and behaviors. Indeed, information appreciated by users now may not remain the same after a moment. Besides, trend events attract users' attention at a specific moment and are no more important after a while. Thus, Social IR systems should be adapted to this evolution. The same as for Social IR systems, the freshness of the information is essential in recommendation systems. So, to enhance recommendation quality, temporal factor should be considered.

Furthermore, positive relations like friends and followers were used and they were proved to have a significant impact in social IR and recommendation. However, negative relations like unfollow in Twitter, dislike on Facebook and Youtube are not taken into account in many works yet [9]. Distrust is also a significant negative relation. This relevant social content is still challenging due to the complexity of having access to negative relations. Moreover, this is a critical issue since obtaining such kind of information is difficult, and analyzing it is also complex. Besides, negative signals are considered provoking and people try to keep them hidden. In [29], authors try to explore the dissolution of follow behavior in Twitter. This study confirms that relational motivations mostly impact the keeping of following relation in Twitter.

Social approaches present certainly some limitations, like the construction of a complete dataset that provides exactly all the information needed. Even information found are very prominent, they can be not suitable for the research's goal. This problem arises specially when constructing the user profile, since not all users provide complete information about themselves. To resolve this issue, some authors propose to combine the available information with the social network graph in order to predict missing information [1].

Another big challenge is to map user's accounts across social networks [32]. In fact, users do not use the same identity in different social networks. That's why it would be so rewarding to collect the user's information from different accounts. 
In [32], authors propose a framework that discovers all different profiles of the same user, providing several inter-social network functionalities. An unsupervised method of linking users across multiple online communities is proposed [21], by disambiguating users provided with the same username.

\section{Proposed recommendation approach}

In this section, we describe our social recommender system that tackles some limits of the existing approaches. In fact, we present in this paper our general proposed approach and we begin by detailing the first step. The other steps will be the subject of future works.

\subsection{General description of our approach}

Our system incorporates social content in a recommender process. In fact, our approach is composed of three steps:

- The identification, extraction and analysis of information gathered from social networks. We collect social information related to users. This social content will be analyzed, in order to build the enhanced social user profile.

- After building social user profiles, we calculate social properties for each user, based on the social content contained in his profile, such as popularity and expertise. We also intend to investigate the temporal aspect to evaluate the freshness of users' social activities. We will then build similar users' groups using clustering algorithms to identify users who may have common or similar preferences [15].

- The third step consists in an exploitation of users' clusters to recommend relations based on their similarities. Furthermore, we will use the temporality of social content to predict the evolution of similar users. Unfollow social content will also be used, in order to analyze its impact on relations' recommendation.

In Figure 1, we present a general description of our proposed social recommender process 


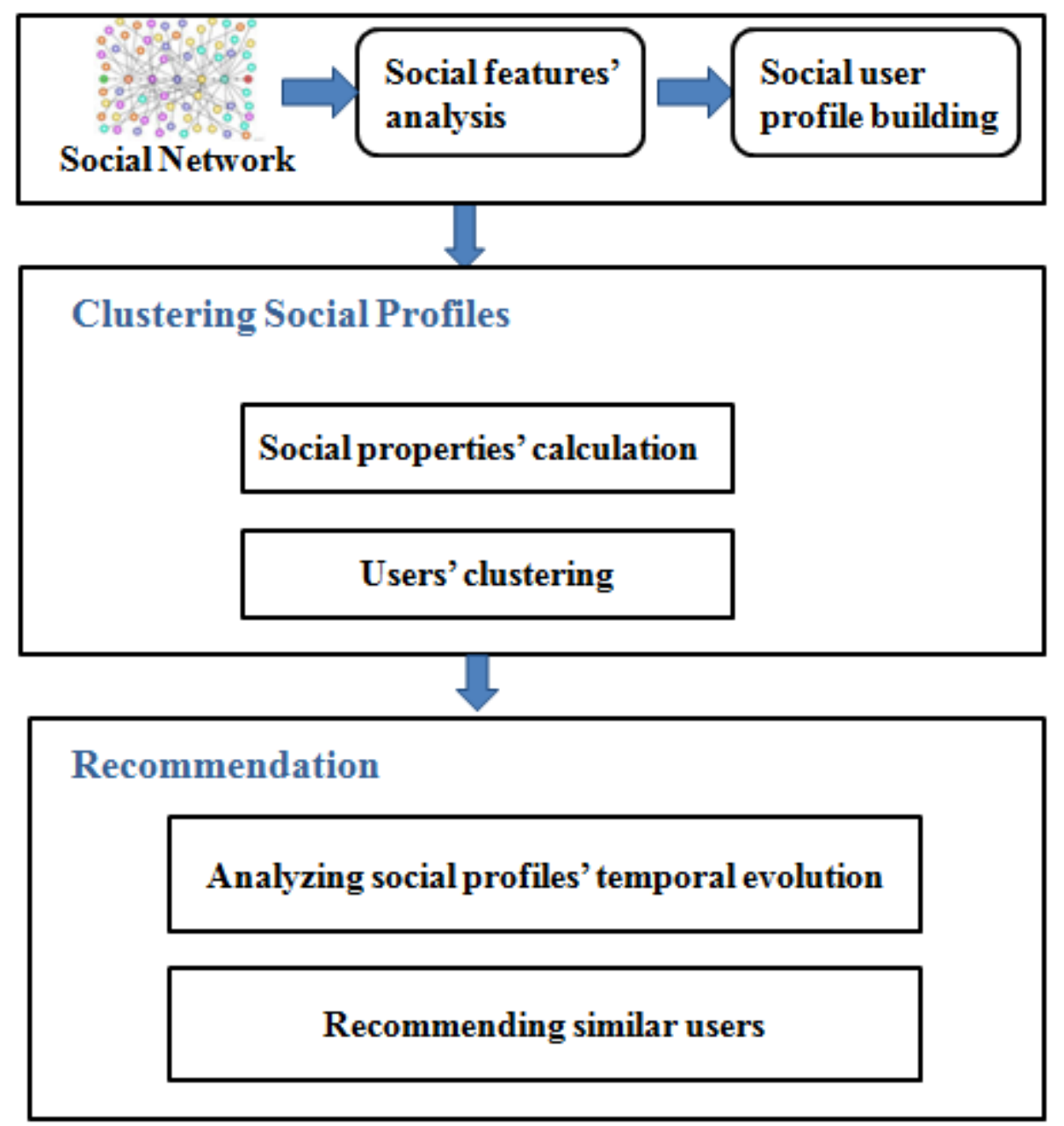

Figure 1. Social Recommender process [15]

\subsection{First step: social user profile building}

To present more details, we started by the first step and we built a temporal and polarity-aware social user profile model [15]. It is a multidimensional social user profile that takes into account temporal aspect of social content and polarized social relations. 
Our social user profile model is a selection of social information reflecting his interests, preferences and experience.

To prepare social information to be used in our social user profile model, we start by extracting social data. There are two possibilities to have access to social information. We can use social networks' API to extract information dynamically, or predefined datasets containing the data needed. We then start a data cleaning and filtering process. In fact, social content extracted should be filtered by eliminating stop words and choosing information suitable to our work. Then, we analyze social data by selecting information to be finally kept and integrated in the user profile. We also calculate several social indicators like the number of user's friends, followers, like and dislike mentions, shares and hashtags. Figure 2 describes this pre-processing task.

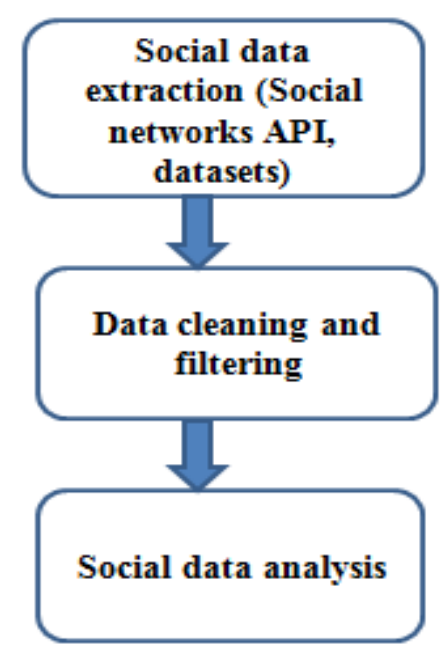

Figure 2. Social information pre-processing

We propose a multidimensional modeling of user profiles which is specially based on the polarized and dynamic social information. In this work we consider the following five dimensions:

Social user profile (Social information, Personal information, Preferences, Experience, Historical information).

\section{Social information}

This dimension is the most important, given the dynamicity of the information it contains, its source and freshness. It is composed of multiple types of social 
information; notably tweets and shared statuses, like and dislike mentions and social relations.

Our social user profile is polarized and temporal. Indeed, we consider not only positive relations like friendship or following relations, but also negative relations and interactions. Particularly, we consider dislike mentions, and focus on unfollow relation on Twitter. Moreover, the temporal aspect of each social feature or interaction is considered; so that we can follow the user profile evolution and predict similar users' profiles evolution.

\section{Personal Information}

Personal information dimension contains general information describing the user, such as his name, age and location.

\section{Preferences}

This dimension includes the interests and preferences of the user. It contains the fields that seem to interest the user. It brings together explicit and implicit interests obtained by a dynamic analysis of the social content of the user.

\section{Experience}

The experience dimension contains information about the user's affiliation, work, scientific or social contributions and domain of expertise.

\section{Historical Information}

Historical information is a dynamic and temporal dimension that invokes users' activities, by monitoring changes in his social interactions.

\section{Conclusion}

In this paper, a review of different aspects of Social IR and recommendation is proposed. We presented a classification of Social approaches into main categories, based on social information used. We also posed a study of Social recommendation systems. Then, we referred to user profile models proposed in Social IR studies, and specially the social dimension. In this respect, works included in this review reflect how deep the impact of social content in IR and recommendation process is. Furthermore, we described our proposed social recommender process including a social user model building. It is a dynamic and polarity-aware social user profile that considers the temporal evolution of social content and both positive and negative social interactions. As current work, we are working on the implementation and notably the evaluation of the social recommender process, incorporating twitter social content. 


\section{References}

[1] A. Mislove, B. Viswanath, K.P. Gummadi, and P. Druschel, "You are who you know: inferring user profiles in online social networks". In Proceedings of the third ACM international conference on web search and data mining, 2010, pp. 251-260.

[2] B.Chahrazed, M.Géry, and L.Christine, "Integrating user's profile in the auerv model for Social Information Retrieval". In Proceedings of the Eighth International Conference on Research Challenges in Information Science, RCIS, 2014, pp. 1-2.

[3] B.Ismail and B.Mohaned, "Document Priors Based On Time-Sensitive Social Signals". In Proceedings of the European Conference on Information Retrieval, ECIR, 2015, pp. 617-622.

[4] B.J.Lamged, T.L.Lynda, and B.Mohaned, "Featured tweet search : Modeling time and social influence for microblog retrieval". In Proceedings of the Web Intelligence and Intelligent Agent Technology, WI-IAT, 2012, pp. 166-173.

[5] B.R. Mohamed, H. Hakim, and B. Mokrane, "Social networks and information retrievl, how are they converging? A survey, a taxonomy and an analysis of social information retrieval approaches and platforms". In Information Systems Journal, 2016, pp. 1-18.

[6] B. Xu, K. Haewoon, and S.C. Noshir, "Structures of broken ties: exnloring unfollow behavior on Twitter". In Proceedings of the 2013 conference on Computer supported cooperative work conference, 2013, pp. 871-876.

[7] C. Jilin, G. Werner, D. Casey, M. Michael, and G. Ido "Make new friends, but keep the old: recommending people on social networking sites". In Proceedings of the SIGHI Conference on Human Factors in Computing Systems, ACM, 2009, pp. 201 210.

[8] C. Marie-Françoise, O.A. Sirinya, P. André, and S. Florence , "Time-aware Egocentric network-based User Profiling". In Proceedings of the International Conference on Advances in Social Networks Analysis and Mining, ASONAM, 2015, pp. 569-572.

[9] C.Sergiu, O.R.Claudia, and S.A.Ismail, "Can social features help learning to rank youtube videos?" In Proceedings of the International Conference on Web Information Systems Engineering WISE, 2012, pp. 552-566.

[10] D. Amna and K. Mouna, "Information Retrieval Framework based on Social Document Profile”. In CAiSE, 2014.

[11] D. Tchuente, C. Marie-Françoise, J. Nadine, P. André, and S. Florence , "A community-based algorithm for deriving users' profiles from egocentrics networks: experiment on Facebook and DBLP”. In Social Network Analysis and Mining,2013, pp. 667-683.

[12] F. Abel, Q. Gao, G.J. Houben, and K. Tao, "Analyzing temporal dynamics in twitter profiles in online social networks", In Proceedings of the 3rd International Web Science Conference, 2011, pp. 2

[13] G. Adomavicius and A. Tuzhilin, "Toward the next generation of recommender systems: A survey of the state-of-the-art and possible extensions. In IEEE Transactions on Knowledge and Data Engineering, 2005, pp. 734-749.

[14] G. Salton and M. McGill, editors. Introduction to Modern Information Retrieval. 
McGraw-Hill,1983

[15] G. Abir, K. Ferihane, and B.G.Henda. Towards a dvnamic and polaritv-aware social user profile modeling. In Proceedings of the 13th ACS/IEEE International Conference on Computer Systems and Applications AICCSA, 2016.

[16] H. Azza, S. Sahbi, G. Malek, and B.G. Henda, "Personalized Information Retrieval: Application to virtual communities.", In Proccedings of the International Conference on Human Interface and the Management of Information, 2014, pp. 431-438.

[17] H. Keejun, P. Juneyoung, and Y.Y. Mun , "Adaptive and multiple interest-aware user profiles for personalized search in folksonomv: A simple but effective graphbased profiling model”. In Proceedings of the International Conference on Big Data and Smart Computing,BIGCOMP, 2015, pp. 225-231.

[18] H. Meriem, G. Mathias, and B. Michel, "LaHC at INEX 2014: Social Book Search". In Working Notes for CLEF Conference, 2014.

[19] H. John, B. Mike, and S. Barry, "Recommending twitter users to follow using content and collaborative filtering approaches". In Proceedings of the fourth ACM conference on Recommender systems, RecSys, 2010, pp. 199-206.

[20] H. Gao, J. Tang, and X. Liu, "Exploring Temporal Effects for Location Recommendation on Location-Based Social Networks", In Proceedings of the $7^{\text {th }}$ ACM conference on Recommender systems, 2013, pp. 93-100.

[21] J. Liu et al., "What's in a name?: an unsupervised approach to link users across communities". In Proceedings of the sixth ACM international conference on Web search and data mining, 2013, pp. 495-504.

[22] J. Zhang, C. Wang, and J. Wang, "Learning temporal dynamics of behavior propagation in social networks". In Twenty-Eighth AAAI Conference on Artificial Intelligence, 2014, pp. 229-236.

[23] L. Li, Z. Li, Y. Fan, and L. Tao, "Track Modeling and broadening temporal user interest in personalized news recommendation". In Expert Systems with Applications Journal, 2014, pp.3168-3177.

[24] L. Xiang et al., "Temporal recommendation on graphs via long-and short-term preference fusion". In Proceedings of the $16^{\text {th }}$ ACM SIGKDD international conference on Knowledge discovery and data mining, 2010, pp. 723-732.

[25] M.Balabanovicand,Y.Shoham."Fab:Content-based. collaborative recommendation", In Communications of the Association for Computing Machinery ACM, 1997, pp. 66-72.

[26] M.Jamali and M. Ester, "A matrix factorization technique with trust propagation for recommendation in social networks". In Recsys, 2010.

[27] M.Jamali and M. Ester, "Trust Walker: A random walk model for combining Trustbased and Item-based Recommendation". In KDD, 2012.

[28] O.A.Nawel, M.Philippe, and G.Mathias, "Recherche de conversations dans les réseaux sociaux : Modélisation et expérimentations sur Twitter", In CORIA, 2015.

[29] P. Lambert, "Fouille exploratoire de messages publiés sur Twitter pour l'aide à la décision", Phd thesis report, University of Nantes, 2015.

[30] P.Li, B.Wang, W.Jin, J.Nie, S.Zhiwei, and B.He, "Exploring categorization property of social annotations for information retrieval". In Proceedings of the $20^{\text {th }}$ ACM international conference on Information and knowledge management, 2011, pp. 557-562.

[31] P. Massa and P. Avesani, “Trust aware recommender systems”. In Recsys, 2007. 
[32] R. Elie, C. Richard, and D. Albert, "User profile matching in social networks". In Network-Based Information Systems, 2010, pp. 297-304.

[33] R. Nathaneal and J. Andrews, "Personalized Search Engine using Social Networking Activity”. In Indian Journal of Science and Technology, 2015, pp. 301.

[34] S. Bao, X. Wu, B. Fei , G. Xue, Z. Su and Yong Yu . "Optimizing web search using social annotations". In Proceedings of the 16th international conference on World Wide Web. ACM, 2007.

[35] S.E. Robertson and S. Walker. "Some simple effective approximations to the 2poisson model for probabilistic weighted retrieval". In Proceedings of the 17th conference on Research and development in Information Retrieval, SIGIR' '94, 1994, pp. 232-241.

[36] S.G. Esparza, M.P. O’Majhony, and B. Smyth, M. Michael, "Mining the real-time web: a novel approach to product recommendation". In Knowledge-Based Systems, 2012, pp. 3-11.

[37] S.H. Yang, B. Long, A. Smola, N. SAdagopan, Z. Zhang, and H. Zha."Like like alike- Joint friendship and interest propagation in social networks", In Proceedings of the 20th international conference on World wide web, www, 2011, pp 537-546.

[38] X. Haoran, L. Xiaodong, T. Wang, L. Chen, L. Ke, L.W. Fu, C. Yi, L. Qing, and M. Huaqing , "Personalized Search for Social Media via Dominating Verbal Context". In Neurocomputing, 2016, pp. 27-37.

[39] X. Wang, H. Liu, and W. Fan, "Connecting users with similar interests via tag network inference". In Proceedings of the $20^{\text {th }}$ ACM international conference on Information and knowledge management, CIKM, 2011, pp. 1019-1024.

[40] X. Yang, H. Steck, and Y. Liu, "Circle-based recommendation in online social networks". In Proceedings of the 18th ACM SIGKDD international conference on knowledge discovery and data mining, 2012, pp. 1267-1275.

[41] Z. Reza and L. Huan, "Connecting Corresponding Identities across Communities". In ICWSM, 2009. 